\title{
Realistic eye model for Embodied Conversational Agents
}

\author{
Guillaume Gibert* \\ Inserm, U846, 18 Avenue Doyen Lépine, 69500 Bron, France \\ Stem Cell and Brain Research Institute, 69500 Bron, France \\ Université de Lyon, Université Lyon 1, 69003, Lyon, France \\ Catherine J. Stevens ${ }^{\dagger}$ \\ Marcs Institute, University of Western Sydney, Locked Bag 1797, Penrith NSW 2751, Australia
}

\section{Introduction}

The eyes play an essential role during face to face communication. They provide important information about visual attention and turntaking during human-human and human-avatar interaction. In fact, the eye is a complex organ and gaze is only one of its behaviours. The pupil and the cornea present time-varying phenomena that a realistic eye model should take into account. The primary role of the pupil is to regulate the amount of light entering the eye in response to the changing environmental illumination. The pupil dilates in low illumination conditions and constricts in high illumination conditions. A physiologically inspired (time varying) model of pupil light reflex (PLR) was proposed by Pamplona and colleagues [Pamplona et al. 2009]. Another kind of pupillary movements are hippus which correspond to spontaneous oscillations of the pupil diameter under steady conditions of illumination. It is considered as a chaotic system with period doubling [Rosenberg and Kroll 1999]. In fact, the oscillations (with a frequency equal to $0.2 \mathrm{~Hz}$ ) go from chaotic to periodic behaviours and vice-versa. Another eye component is the cornea which is a transparent tissue that covers the iris. On its surface, a reflection of the world surrounding the person appears: this effect is called corneal reflection. Only recently, an anatomically accurate eye model (replicating video of human eye) has been implemented that takes into account this reflection [François et al. 2007] but not tested embedded in an avatar interacting with humans. The aim of this study was to build a 3D eye model for virtual humans replicating in real-time corneal reflection, pupil size variation due to bottom-up processes and the hippus phenomenon.

\section{Eye model}

Each eye model was composed of different 3D objects: an eyeball, a pupil, an iris and a cornea. A webcam mounted on top of the screen displaying the avatar and facing the human partner played the role of the avatar's eyes, ie., provided visual input. Information retrieved from the webcam can help the avatar to sense the physical world and to adapt its pupil size and simulate the corneal reflection phenomenon. Two different images appear on the cornea of each eye. To create an accurate corneal reflection, each eye was processed separately. Two factors were necessary to determine which part of the webcam image needed to be used for each eye: the gaze point and the rigid head motion. Then, the webcam image was cropped around the gaze point. Two separate images (one for the right eye and one for the left eye) were copied/warped onto the 3D cornea objects. A reflection of the world surrounding the avatar can be simulated on its corneas. The pupil diameter of the avatar can be accurately controlled given an estimation of the ambient lighting conditions of the physical world. The average intensity of the grayscale cornea images was computed and used to control the variation of the pupil size via an adapted version of the model of pupil light reflex proposed by Pamplona and colleagues [Pam-

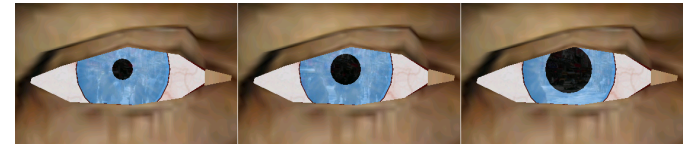

Figure 1: Combined corneal reflection phenomenon and pupil size variation for different scenes viewed by the avatar.

plona et al. 2009]. The simulation of the PLR was performed using Equation 1. The variable $\tau$ controlled the pupil constriction/dilation velocity and was set to $200 \mathrm{~ms}$ for constriction and $600 \mathrm{~ms}$ for dilation. $D_{t}$ corresponded to the ratio of the current corneal image intensity and an intensity threshold.

$$
D_{P L R}=D_{t}\left(1-\exp ^{-\frac{t-t 0}{\tau}}\right)
$$

In addition, the hippus (i.e. a low frequency variation of the apparent pupil diameter) was also implemented in our model. During steady conditions of illumination, the hippus phenomenon was simulated by varying between a period of small random pupillary movements (cahotic behaviour) and a period of oscillating $(0.2 \mathrm{~Hz})$ pupillary movements (periodic behaviour). Combined effects on the avatar's eye can be seen on Figure 1. The inclusion of these behaviours in an embodied conversational agents may increase his presence.

\section{Acknowledgements}

This work was supported by the Thinking Head project (ARC and NH\&MRC TS0669874) and by the SWoOZ project (ANR 11 PDOC 019 01).

\section{References}

François, G., Gautron, P., Breton, G., And Bouatouch, K. 2007. Anatomically accurate modeling and rendering of the human eye. In ACM SIGGRAPH 2007 sketches, ACM, New York, NY, USA, SIGGRAPH '07.

Pamplona, V. F., Oliveira, M. M., and Baranoski, G. V. G. 2009. Photorealistic models for pupil light reflex and iridal pattern deformation. ACM Transactions on Graphics 28, $4,23-40$.

Rosenberg, M. L., And Kroll, M. H. 1999. Pupillary hippus: an unrecognized example of biological chaos. Journal of Biological Systems 7, 1, 85-94.

*e-mail:guillaume.gibert@inserm.fr

†e-mail:kj.stevens@uws.edu.au 